Research Article

\title{
Spectrum Efficiency Optimization for UAV-Based Cognitive Radio Network
}

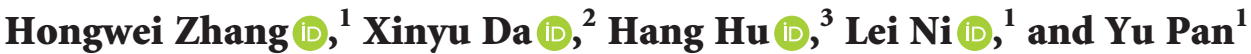 \\ ${ }^{1}$ Graduate School, Air Force Engineering University, Xi'an 710077, China \\ ${ }^{2}$ College of Artificial Intelligence, Yango University, Fuzhou 350015, China \\ ${ }^{3}$ College of Information and Navigation, Air Force Engineering University, Xi'an 710077, China
}

Correspondence should be addressed to Xinyu Da; kgddxy2008@163.com

Received 18 March 2020; Revised 10 June 2020; Accepted 20 June 2020; Published 13 July 2020

Academic Editor: Junwon Seo

Copyright (c) 2020 Hongwei Zhang et al. This is an open access article distributed under the Creative Commons Attribution License, which permits unrestricted use, distribution, and reproduction in any medium, provided the original work is properly cited.

\begin{abstract}
Unmanned aerial vehicle- (UAV-) assisted communication has great potential to provide on-demand wireless services and improve the outdoor link throughput. In this paper, a UAV-based cognitive radio network (CRN) is investigated in which the UAV works as a secondary user (SU). Considering the overlay spectrum sensing mode, the UAV can operate on the licensed spectrum bands of primary user (PU) only when PU is idle. In each working frame structure, both sensing time slot and transmission time slot are analysed in radians. Specifically, our objective is to maximize the spectrum efficiency (SE) of the UAV by jointly optimizing the sensing radian and the number of radians. For the single-radian and multiradian schemes, the dichotomy and alternative iterative optimization (AIO) algorithm are proposed to solve the SE optimization problem. Simulation results show that the proposed multiradian cooperative spectrum sensing (CSS) scheme can achieve better performance on ensuring the quality-of-service (QoS) of the PU, and it can significantly enhance the SE of the UAV especially in the severe channel environments.
\end{abstract}

\section{Introduction}

Recently, unmanned aerial vehicles (UAVs) have remarkably gained much popularity in various communication scenarios due to their advantages of high maneuverability and flexibility [1]. Generally, UAVs are initially designed for military operations, tracking and surveillance, fire control, and other applications [2, 3], most of which are dangerous and even impossible for human operators. Due to the gradual enrichment of their functions and the continuous development of sensor technology, the UAVs equipped with sensors and communication equipment can be applied in various fields. With the help of these new technologies, the UAVs are widely used in many complicated tasks. Compared with terrestrial communication, UAV communication has less path loss when transmitting data; thus the UAV will have a broad application prospect in communication technology [4]. However, the application of UAV communication also faces many challenges, and spectrum shortage is one of the key issues. Due to the rapid development of 5G network [5], device-to-device (D2D) technology [6], and Internet of Things (IoT) [7], the spectrum demand is growing rapidly. The UAV generally operates on IEEE S-band, IEEE L-band, and industrial, scientific, and medical (ISM) frequency bands, and these spectrum resources have been occupied by many wireless networks (such as Wi-Fi, Bluetooth, and IEEE 802.15.4 network) [8]. This makes the problem of spectrum shortage more serious. Hence, it is of great significance to investigate the optimization of spectrum efficiency (SE).

Cognitive radio (CR), as a technique to improve the SE, enables the secondary users (SUs) to utilize the licensed spectrum of the primary users (PUs) [9]. The SU can detect the spectrum hole by checking the channel conditions of the PU through spectrum sensing. In overlay mode, only when PU is detected to be free can the SUs access the PU's 
spectrum. The SU will interfere with the normal operation of PU if missed detection happens in the sensing process. Therefore, the detection probability of spectrum sensing should be improved to ensure the quality-of-service (QoS) of the PU [10]. Energy detection has been widely used in cognitive wireless networks as an effective spectrum sensing method, which can determine the sensing results by comparing the energy statistic of received signal with the sensing threshold [11]. However, the influence of channel fading and noise interference will result in poor detection performance. Therefore, cooperative spectrum sensing (CSS) is proposed to improve the sensing performance [12]. In CSS, multiple local sensing results are comprehensively analysed to obtain the final decision by the fusion centre (FC) using "logicAND" rule, "logic-OR" rule, and " $n$-out-of- $K$ " rule [13]. The CSS can improve sensing performance and will consequently increase SE by optimizing the secondary link throughput. However, if more time is consumed in CSS process, the transmission time will be reduced and the throughput may not be increased accordingly. Therefore, the sensing time should be optimized to maximize the secondary link throughput [14].

The UAVs are less affected by fading and shadowing in air-to-ground (A2G) channel and can achieve better SNR of the received signal. Therefore, some recent studies have focused on the SE optimization of UAV-enabled cognitive radio network (CRN). It is pointed out in [15] that the CR technology integrated with UAV can bring significant SE benefits in the scenario which is deployed with large-scale UAVs. In [16], an uplink MIMO-CR system is investigated, where the PU and the SU communicate with the closest base station (BS) through the same UAV relay and multiple access channels, and the SE of SU can be maximized by optimizing power allocation. In [17], the transmission rate performance of the A2G link is simulated and analysed by establishing an accurate A2G link channel model. In [18], the system parameters of cooperative sensing in cognitive UAV network are optimized to improve the energy efficiency and maximize the SE of SU. However, the SE optimization of UAV-enabled cognitive radio network (CRN) is still in infancy and few literatures were presented. The technique of this novel system still needs extensive and detailed theoretical research.

Aiming at the problem of spectrum resource shortage in UAV Communication Network, in this paper, a UAV-based CRN is established, in which the UAV monitors the ground in a circular flight and transmits data to BS during flight. Moreover, the SE optimization problem of the UAV in A2G channel is analysed by optimizing the sensing radian subject to the QoS constraint of PU. Then an SE optimization scheme for multiradian CSS is proposed to improve the sensing performance. The main contributions of our work are as follows:

(1) A UAV-based overlay CRN is established to investigate the SE optimization of the UAV in A2G channel on the premise of guaranteeing the QoS of PU. In this model, the UAV flies circularly and performs spectrum sensing and data transmission in each frame, and the sensing radian is optimized to maximize the SE and minimize the data transmission time of the secondary UAV link.

(2) The joint optimization of the sensing radian and the number of radians is achieved by our proposed AIO algorithm to maximize the average throughput of the UAV-based CRN.

(3) The performances of the system are analysed by numerical simulation results to evaluate the SE of the UAV. Then, the proposed AIO algorithm is compared with the single-radian optimization (SRO) algorithm in [19]. Simulation results show that sensing radian optimization can make the UAV achieve expected communication performance, while the multiradian CSS technology performs better on improving the SE of UAV and guaranteeing the QoS of PU, especially in the severe channel environments.

The rest of this paper is organized as follows. In Section 2, the UAV-based CRN model in A2G channel is established. In Section 3, the single- and multiradian schemes for SE optimization are proposed. The simulation results are presented in Section 4, while our conclusions are drawn in Section 5 .

\section{System Model}

As shown in Figure 1, we consider a UAV-based CRN, where the UAV communicates with ground users as a SU and monitors the ground through cameras and sensor nodes to detect fire detection, illegal intrusion, and other emergencies in time. The distance between the PU and the BS is denoted as $R_{P}$, and the flight radius of the UAV is denoted as $R_{S}$. The $\mathrm{BS}$ is considered to be located in the centre of this network, and the UAV flies circularly with flight speed given as $v$. In addition, the flight altitude is denoted as $H$, which is supposed to be fixed in this paper. The UAV performs periodic circular flight around the BS, and each flight cycle contains $l$ flight time slots, which are divided into sensing time slot and transmission time slot. When the PU is detected to be idle, the UAV can access the licensed spectrum and transmit data in the transmission time slot. It is assumed that the UAV transmits $M$ bits of data to the BS within $T_{a}$ seconds; that is,

$$
T_{a}=\frac{M}{\mathbb{R}}
$$

where $T_{a}$ changes dynamically according to the bit rate of SU given by $\mathbb{R}$.

In this model, in order to facilitate the investigation of the circular flight characteristics, both sensing time slot and transmission time slot are analysed in radians, which are defined as sensing radian and transmission radian. The radian corresponding to one flight time slot is assumed as $\mathrm{B}$, sensing radian is $\theta$, and transmission radian is $\mathrm{B}-\theta$. Therefore, the sensing time $t_{s}$ can be given by

$$
t_{s}=\frac{R_{S} \theta}{v}
$$




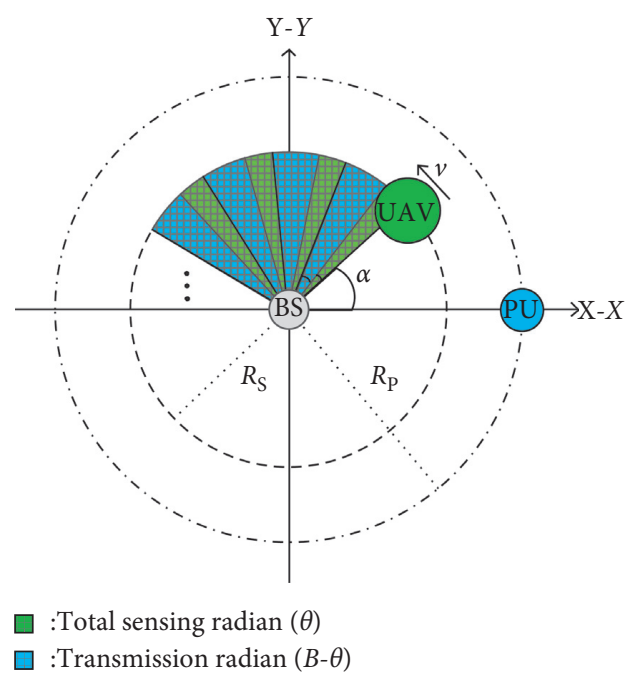

(a)

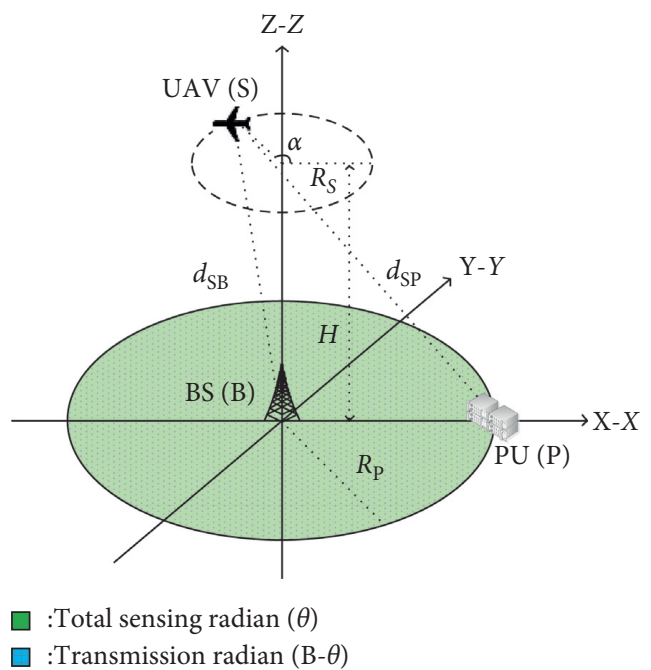

(b)

FIgURE 1: The UAV-based CRN model. (a) Two-dimensional model of UAV-based CRN. (b) Three-dimensional model of UAV-based CRN.

The positions of the BS, the $\mathrm{PU}$, and the UAV are as shown in Figure 1, which are denoted as B, P, and S, respectively. The angle between the UAV and $x$-axis is denoted as $\alpha$, and the distance between the UAV and the PU is given by

$$
d_{\mathrm{sp}}=\sqrt{H^{2}+R_{S}^{2}+R_{P}^{2}-2 R_{S} R_{P} \cos \alpha} .
$$

The distance between the UAV and the BS is expressed as

$$
d_{\mathrm{SB}}=\sqrt{R_{S}^{2}+H^{2}}
$$

In this model, $h_{\mathrm{SB}}, h_{\mathrm{SP}}$, and $h_{\mathrm{PB}}$ are used to represent the channel gain between the UAV and the BS, between the $\mathrm{UAV}$ and the PU, and between the PU and the BS, respectively. Compared to the channel coherence time, the transmission time is relatively long, so we focus on the average statistics of the channel. It is impossible to investigate the instantaneous channel realization of the framework and the system performance of network statistics since the UAVs' flying time, commonly in seconds, is very large compared to the channel coherence time, which is usually measured in milliseconds [20]. Therefore, we only consider the large-scale path loss in this paper. The channel gain expression can be derived as follows:

$$
h_{i j}\left(d_{i j}\right)=\frac{1}{\sqrt{L_{i j}\left(d_{i j}\right)}}
$$

where $h_{i j}$ represents the channel gain of three different links and $L_{i j}\left(d_{i j}\right)$ is the path loss between $i$ and $j$, where $i \in\{S, P\}$ and $j \in\{P, B\}$. Two types of channel models are investigated in this system:

(1) Ground-to-ground (G2G) channel, such as $h_{\mathrm{PB}}$ in the model. The G2G channel between PU and BS is non-line-of-sight (NLoS) channel because of the long distance and obstructions between PU and BS.
(2) Air-to-ground (A2G) channel, such as $h_{\mathrm{SB}}$ and $h_{\mathrm{SP}}$ in the model. The probability of the line-of-sight (LoS) link in A2G channel is denoted as $P_{i j}^{\mathrm{LoS}}$, which is dependent on the elevation angle between the UAV and ground node and environmental characteristics.

The probability of the LoS link is given by [21]

$$
P_{i j}^{\mathrm{LoS}}=\frac{1}{1+\omega_{1} \exp \left(-\omega_{2}\left((180 / \pi) \beta_{i j}-\omega_{1}\right)\right)},
$$

where $\beta_{i j}$ is the elevation angle between the UAV and ground node, $\omega_{1}$ and $\omega_{2}$ are the environmental characteristic parameters, and the average path loss of G2G channel and A2G channel can be obtained as

$$
L_{i j}\left(d_{i j}\right)= \begin{cases}L_{i j}^{\mathrm{NLoS}}\left(d_{i j}\right) & \mathrm{G} 2 \mathrm{G}, \\ \mathrm{P}_{\mathrm{ij}}^{\mathrm{LoS}} L_{i j}^{\mathrm{LoS}}\left(d_{i j}\right)+\left(1-\mathrm{P}_{\mathrm{ij}}^{\mathrm{LoS}}\right) L_{i j}^{\mathrm{NLoS}}\left(d_{i j}\right) & \mathrm{A} 2 \mathrm{G},\end{cases}
$$

where $L_{i j}^{\mathrm{LoS}}$ and $L_{i j}^{\mathrm{NLoS}}$ represent the average path loss in the LoS and the NLoS links, respectively:

$$
L_{i j}^{X}\left(d_{i j}\right)=20 \log _{10}\left(\frac{4 \pi f d_{i j}}{C}\right)+\zeta_{x}, X \in\{\operatorname{LoS}, \text { NLoS }\},
$$

where $C$ is the speed of light, $f$ is the carrier frequency, and $\zeta_{x}$ is the average additional propagation loss of the LoS and the NLoS links.

The energy detection method is used to sense the PU's status, and the sensing result is determined by comparing the test statistic with the energy detection threshold $\lambda$. It is assumed that the UAV receives the following signals:

$$
y(m)=\mu s(m) h_{i j}(m)+n(m), \quad m=1,2, \ldots, D,
$$

where $\mu$ is the status parameter of the PU and $\mu=1$ and $\mu=0$ represent the cases where the true status of PU is present and absent, respectively, $s(m)$ is the signal from the 
primary transmitter, $h_{\mathrm{ij}}(m)$ is the channel gain between nodes $i$ and $j, n(m)$ is the noise at the UAV, and $D$ is the number of sampling points, which is given by $D=t_{s} f_{s}$, where $f_{s}$ is the sampling frequency. The test statistic of $y(m)$ is given as

$$
E(y)=\frac{1}{D} \sum_{m=1}^{M}|y(m)|^{2}
$$

This statistic obeys Gaussian distribution when the value of $D$ is large enough; then the false alarm probability and the detection probability can be computed by [22]

$$
\begin{gathered}
P_{f}(\lambda, \theta)=P_{r}(E(y)>\lambda \mid \mu=0)=\mathbb{Q}\left(\left(\frac{\lambda}{\sigma_{n}^{2}}-1\right) \sqrt{\theta \frac{R_{S} f_{S}}{v}}\right), \\
P_{d}(\lambda, \theta)=P_{r}(E(y)>\lambda \mid \mu=1) \\
=\mathbb{Q}\left(\left(\frac{\lambda}{\sigma_{n}^{2}}-\gamma-1\right) \sqrt{\theta \frac{R_{S} f_{S}}{v(2 \gamma+1)}}\right)
\end{gathered}
$$

where $\sigma_{n}^{2}$ is the noise power, $\gamma$ is the sensing signal-to-noise ratio (SNR), which is given by $\gamma=\left(P_{P}\left|h_{\mathrm{SP}}\right|^{2} / \sigma_{n}^{2}\right), P_{p}$ is the transmission power of the PU, and $\mathbb{Q}(x)$ is the complementary distribution function of the standard Gaussian given as [22]

$$
\mathbb{Q}(x)=\frac{1}{\sqrt{2 \pi}} \int_{x}^{+\infty} \exp \left(-\frac{u^{2}}{2}\right) d u .
$$

The target detection probability $\bar{P}_{d}$ is usually preset to ensure the sensing performance; then the false alarm probability can be obtained by combining (11) and (12):

$$
P_{f}=\mathbb{Q}\left(\varepsilon+\gamma \sqrt{\theta \frac{R_{S} f_{s}}{v}}\right),
$$

where $\varepsilon=\sqrt{2 \gamma+1} \mathbb{Q}^{-1}\left(\bar{P}_{d}\right)$. Similarly, for a given false alarm probability $\bar{P}_{f}$, the detection probability can be obtained by combining (11) with (12):

$$
P_{d}=\mathbb{Q}\left(\frac{1}{\sqrt{2 \gamma+1}}\left(\mathbb{Q}^{-1}\left(\bar{P}_{f}\right)-\gamma \sqrt{\theta \frac{R_{S} f_{s}}{v}}\right)\right) .
$$

\section{Optimization of SE}

3.1. Optimization of SE in Single Radian. The optimization of the UAV's sensing performance and achievable average throughput are investigated in this section. First of all, from (5) and (6), it can be seen that the probability of the LoS link is highly related to the location of the UAV, because the flight height and the flight radius of the UAV will change the distance and elevation angle between the UAV and ground node. Therefore, the values of the flight height and the flight radius are fixed in this paper; only the impact of the flight speed on sensing radian is analysed to investigate the relationship between sensing radian and throughput. In addition, it will be proved that there exists one optimal sensing radian under the premise of ensuring the QoS of the PU.

The UAV is assumed to perform spectrum sensing first and then transmits data to the BS if the PU is detected to be free. The transmission rate of the UAV is denoted as $\pi_{0}$ when the PU is idle, which is given by $\pi_{0}=\log \left(1+\left(P_{S}\left|h_{\mathrm{SB}}\right|^{2} / \sigma_{n}^{2}\right)\right)$, where $P_{S}$ is the transmission power of the UAV. When the $\mathrm{PU}$ is busy, the transmission rate of the UAV is defined as $\pi_{1}$, which is given by $\pi_{1}=\log \left(1+\left(P_{S}\left|h_{\mathrm{SB}}\right|^{2} / \sigma_{n}^{2}+P_{P}\left|h_{\mathrm{PB}}\right|^{2}\right)\right)$, where $P_{P}\left|h_{\mathrm{PB}}\right|^{2}$ represents the interference power of the PU received by $\mathrm{BS}$. The parameter $\mu$ is used to indicate the absence or existence of the PU, the probability that PU does not exist is given by $P_{r}(\mu=0)$, and the probability that $\mathrm{PU}$ exists is given by $P_{r}(\mu=1)$.

Two cases are considered when the UAV utilizes the frequency band of the PU.

Case 1: the PU's true status is idle, and no false alarm happens when the UAV performs spectrum sensing. In this case, the transmission rate of the secondary UAV link is $(B-\theta / B) \pi_{0}$.

Case 2: the PU's true status is busy, but missed detection happens. In this case, the transmission rate of the secondary UAV link is $(B-\theta / B) \pi_{1}$.

The probabilities of case 1 and case 2 are given as (1$\left.P_{f}\right) P_{r}(\mu=0)$ and $\left(1-P_{d}\right) P_{r}(\mu=1)$, respectively; then the throughput for the two cases can be derived as follows:

$$
\left.\begin{array}{l}
\mathbb{R}_{0}(\lambda, 0)=\frac{B-\theta}{B} P_{r}(\mu=0)\left(1-P_{f}(\lambda, 0)\right) \pi_{0} \\
\mathbb{R}_{1}(\lambda, 0)=\frac{B-\theta}{B} P_{r}(\mu=1)\left(1-P_{d}(\lambda, 0)\right) \pi_{1}
\end{array}\right\} .
$$

The average throughput of the UAV link can be expressed as

$$
\mathbb{R}(\theta)=\mathbb{R}_{0}(\lambda, \theta)+\mathbb{R}_{1}(\lambda, \theta)
$$

Hence, the time for UAV to transmit $M$ bits of data to BS is given by

$$
T_{a}=\frac{M}{\mathbb{B R}(\theta)}
$$

where $\mathbb{B}$ is the bandwidth.

In addition, it can be obtained from $(13)$ that $\mathbb{Q}(x)$ is a monotonic decreasing function of $x$. For a given target detection probability $\bar{P}_{d}$, the false alarm probability decreases when the sensing radian increases. This gives UAV more opportunities to access the idle spectrum. The purpose of SE optimization is to find the optimal sensing radian of the UAV to maximize the average throughput of the UAV. The optimization problem can be formulated as

$$
\begin{array}{ll}
\max _{\theta} & \mathbb{R}(\theta)=\mathbb{R}_{0}(\lambda, \theta)+\mathbb{R}_{1}(\lambda, \theta), \\
\text { s.t. } & P_{d}(\lambda, \theta) \geq \bar{P}_{d}, \\
& 0 \leq \theta \leq B .
\end{array}
$$


Generally, if the detection probability is not less than $\bar{P}_{d}$, the $\mathrm{PU}$ is defined as being sufficiently protected. In practice, the target detection probability $\bar{P}_{d}$ is close to but less than 1 . For example, in IEEE802.22 WLAN, the target detection probability threshold is $\bar{P}_{d}=0.9$ [22]. It should be noted that if the PU requires $100 \%$ protection, its frequency band will not be available to be shared with UAV. In addition, if the active probability of the PU denoted as $P_{r}(\mu=1)$ is small, for example, $P_{r}(\mu=1) \leq 0.3$, spectrum resources can be effectively utilized by exploring the spectrum holes from the perspective of green economy. Since $\pi_{0}>\pi_{1}, P_{r}(\mu=0)>P_{r}(\mu=1)$, and $\left(1-P_{f}\right)>\left(1-\bar{P}_{d}\right)$, the first term on the right of (19) dominates the average throughput. The approximate value of the average throughput is denoted as $\mathbb{R}_{A}$, and the optimization problem can be simplified as follows:

$$
\begin{array}{ll}
\max _{\theta} & \mathbb{R}_{A}(\theta)=\mathbb{R}_{0}(\lambda, 0), \\
\text { s.t. } & P_{d}(\lambda, \theta) \geq \bar{P}_{d}, \\
& 0 \leq \theta \leq B .
\end{array}
$$

So the time for UAV to transmit $M$ bits of data to BS is approximated as $T_{a}=\left(M / \mathbb{B R}_{A}(\theta)\right)$.

It is supposed that $\lambda_{0}$ is the energy detection threshold, which satisfies $P_{d}\left(\lambda_{0}, \theta\right)=\bar{P}_{d}$. For an energy detection threshold $\lambda_{1}$, if $\lambda_{1}<\lambda_{0}$, we can achieve $P_{d}\left(\lambda_{1}, \theta\right)>\bar{P}_{d}=P_{d}\left(\lambda_{0}, \theta\right)$ and $P_{f}\left(\lambda_{1}, \theta\right)>P_{f}\left(\lambda_{0}, \theta\right)$; then $\mathbb{R}_{0}\left(\lambda_{1}, \theta\right)<\mathbb{R}_{0}\left(\lambda_{0}, \theta\right)$ and $\mathbb{R}_{1}\left(\lambda_{1}, \theta\right)<\mathbb{R}_{1}\left(\lambda_{0}, \theta\right)$ can be obtained according to (16). Therefore, for optimization problems (19) and (20), when the energy detection threshold is $\lambda_{0}$, the average throughput can be maximized.

When the detection probability is $P_{d}=\bar{P}_{d}$, the optimization problem (20) can be reformulated as

$$
\max _{\theta} \mathbb{R}_{A}(\theta)=\left(1-\frac{\theta}{B}\right) P_{r}(\mu=0)\left(1-P_{f}(\theta)\right) \pi_{0}
$$

\section{s.t. $0 \leq \theta \leq B$,}

Then, we will analyse the property of the objective function. Taking the first derivative of $P_{f}(\theta)$ with respect to $\theta$, we can obtain that

$$
\frac{d P_{f}(\theta)}{d \theta}=-\frac{\gamma \sqrt{R_{S} f_{s}}}{2 \sqrt{2 \pi \theta v}} \exp \left(-\frac{1}{2}\left(\varepsilon+\gamma \sqrt{\theta \frac{R_{S} f_{s}}{v}}\right)^{2}\right) .
$$

Obviously, it can be obtained that $\left(d P_{f}(\theta) / d \theta\right)<0$ since sensing radian $\theta>0$; thus $P_{f}(\theta)$ decreases monotonically with the increase of $\theta$. Furthermore, we can obtain that $\mathbb{Q}(x) \leq 0.5$ when $x \geq 0$ from the property of $\mathbb{Q}(x)$. Therefore, we have $\varepsilon+\gamma \sqrt{\theta\left(R_{S} f_{s} / v\right)} \geq 0$ when $P_{f}(\theta) \leq 0.5$. It can be proved that $\left(d P_{f}(\theta) / d \theta\right)$ monotonically increases with $\theta$ and $P_{f}(\theta)$ is convex when $P_{f}(\theta) \leq 0.5$.

Theorem 1. There exists only one optimal $\theta \in[0, B]$ that maximizes $\mathbb{R}_{A}(\theta)$.

Proof. Taking the first derivative and the second derivative of $\mathbb{R}_{A}(\theta)$ with respect to $\theta$, we can obtain that

$$
\frac{d \mathbb{R}_{A}(\theta)}{d \theta}=\left(-\left(1-\frac{\theta}{B}\right) \frac{d P_{f}(\theta)}{d \theta}-\frac{1}{B}\left(1-P_{f}(\theta)\right)\right) \pi_{0} P_{r}(\mu=0) .
$$

$$
\frac{d^{2} \mathbb{R}_{A}(\theta)}{d^{2} \theta}=\left(-\left(1-\frac{\theta}{B}\right) \frac{d^{2} P_{f}(\theta)}{d^{2} \theta}-\frac{2}{B} \frac{d P_{f}(\theta)}{d \theta}\right) \pi_{0} P_{r}(\mu=0) .
$$

From (22) and (24), it is derived that $\left(d^{2} \mathbb{R}_{A}(\theta) / d^{2} \theta\right)<0$ when $P_{f}(\theta) \leq 0.5$, and $\mathbb{R}_{A}(\theta)$ is a concave function. Then, the bound of $\left(d \mathbb{R}_{A}(\theta) / d \theta\right)$ with $\theta \in[0, B]$ can be achieved as follows:

$$
\begin{aligned}
& \lim _{\theta \longrightarrow \mathrm{B}} \frac{d \mathbb{R}_{A}(\theta)}{d \theta}<\pi_{0} P_{r}(\mu=0)\left(-\frac{1}{B}+\frac{1}{B} \mathbb{Q}(\varepsilon)\right)<0, \\
& \lim _{\theta \longrightarrow 0} \frac{d \mathbb{R}_{A}(\theta)}{d \theta}=+\infty .
\end{aligned}
$$

In the derivation of equation (25), the property that $\mathbb{Q}(x)$ is a decreasing function and is upper bounded by 1 has been used. Note that equations (25) and (26) are used to prove that function $\mathbb{R}_{A}$ increases first and then decreases within interval $[0, B]$. Hence, there exists a unique value of $\theta \in[0, \mathrm{~B}]$ that makes $\left(d \mathbb{R}_{A}(\theta) / d \theta\right)=0$, which is defined as $\theta^{*}$; thus $\mathbb{R}_{A}$ will achieve the maximum when $\theta=\theta^{*}$. The Algorithm 1 to achieve $\theta^{*}$ is stated as follows.

Two kinds of sensing scenarios for the UAV are considered when investigating the optimal sensing radian:

Scenario 1: in the case of static sensing, the effect of changing $d_{\mathrm{SP}}$ on $\gamma$ can be ignored during the UAV flight, and $\gamma$ is considered to be constant during the sensing process because of the small sensing radian of the UAV. Therefore, the sensing process can be considered as static sensing in one frame [23].

Scenario 2: in the case of dynamic sensing, UAV flies around the BS in a circle with $l$ spectrum sensing frames, and the radian of each frame is $B=(2 \pi / l)$. In addition, the optimal sensing radians in each frame are different, since $\gamma$ varies with the flight position of UAV in each frame.

3.2. Optimization of SE in Multiradian CSS. In this section, a multiradian CSS scheme that combines multiple local sensing results is proposed, and the sensing slot is divided into multiple contiguous mini slots in this case. The UAV has different sensing paths in different mini slots, and the cooperative diversity gain of the UAV can be obtained by aggregating sensing information from multiple sensing paths. The UAV obtains local decision in each mini slot, and the final decision can be achieved by fusing all local decision results through the FC, as shown in Figure 2. All the local detection results can be obtained synchronously by the UAV when its speed is fast, so the UAV's multiradian spectrum sensing can be regarded as CSS.

It is supposed that the total sensing radian in each frame is composed of multiple mini sensing radians and each mini 


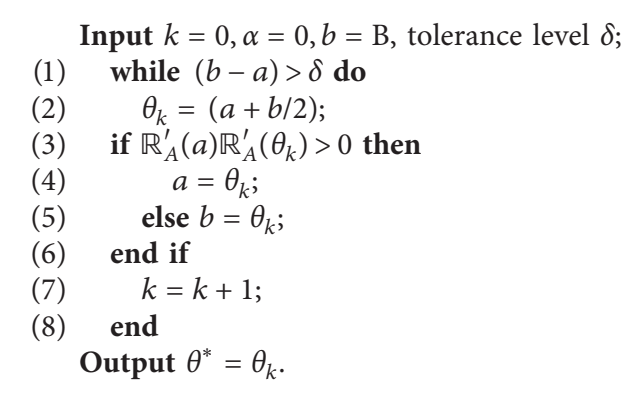

Algorithm 1: Optimization of $\theta$ that maximizes $\mathbb{R}_{A}(\theta)$.

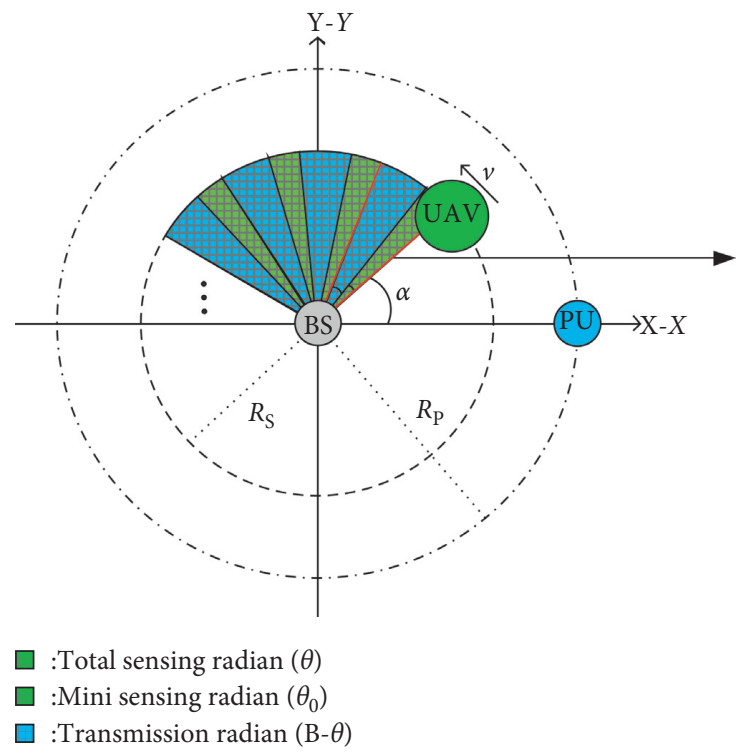

(a)

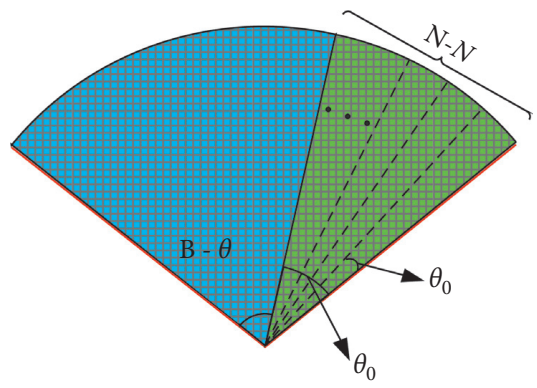

:Total sensing radian $(\theta)$

:Mini sensing radian $\left(\theta_{0}\right)$

:Transmission radian $(\mathrm{B}-\theta)$

FIGURE 2: Multiradian CSS frame structure. (a) Two-dimensional model of UAV-based CRN; (b) CSS frame structure of UAV-based CRN.

sensing radian is $\theta_{0}$. Let $N$ denote the number of mini sensing radians; then the total sensing radian for each frame is $\theta=N \theta_{0}$, and the transmission radian for each frame is $B-N \theta_{0}$. The "OR" rule is adopted by FC to make decision by combining all local decisions of each mini sensing radian. In "OR" rule, if any local sensing result has shown the presence of the $\mathrm{PU}$, then the final decision is that the $\mathrm{PU}$ is present. The false alarm probability $P_{f}\left(\lambda, \theta_{0}\right)$ and the detection probability $P_{d}\left(\lambda, \theta_{0}\right)$ of each mini sensing radian have been given in (11) and (12). The expression of cooperative false alarm probability and cooperative detection probability of multiradian are derived as follows:

$$
\begin{gathered}
Q_{f}=1-\left(1-P_{f}\right)^{N}, \\
Q_{d}=1-\left(1-P_{d}\right)^{N} .
\end{gathered}
$$

The target detection probability of CSS is set as $\bar{Q}_{d}$ to ensure the final sensing performance, and the local target detection probability $\bar{P}_{d}$ can be derived as
$\bar{P}_{d}=1-\left(1-\bar{Q}_{d}\right)^{(1 / N)}$. From (14), (27), and (28), the relationship between $Q_{f}$ and $\bar{Q}_{d}$ can be given as

$$
Q_{f}=1-\left(1-\mathbb{Q}\left(\sqrt{2 \gamma+1} \mathbb{Q}^{-1}\left(1-\left(1-\bar{Q}_{d}\right)^{\frac{1}{N}}\right)+\gamma \sqrt{\theta_{0} \frac{R_{S} f_{s}}{v}}\right)\right)^{N} .
$$

The throughput of UAV in two cases can be calculated as follows:

$$
\mathbb{R}_{0}\left(\lambda, \theta_{0}, N\right)=\frac{B-N \theta_{0}}{B} P_{r}(\mu=1)\left(1-P_{f}\left(\lambda, \theta_{0}\right)\right)^{N} \pi_{0},
$$

$$
\mathbb{R}_{1}\left(\lambda, \theta_{0}, N\right)=\frac{B-N \theta_{0}}{B} P_{r}(\mu=1)\left(1-P_{d}\left(\lambda, \theta_{0}\right)\right)^{N} \pi_{1}
$$

In this section, the mini sensing radian $\theta_{0}$ and the number of radians $N$ are jointly optimized to maximize the 
average throughput of the secondary link. The mathematical expression of this optimization problem is formulated as

$$
\begin{array}{ll}
\max _{\theta_{0}, N} & \mathbb{R}\left(\theta_{0}, N\right)=\mathbb{R}_{0}\left(\lambda, \theta_{0}, N\right)+\mathbb{R}_{1}\left(\lambda, \theta_{0}, N\right), \\
\text { s.t. } & Q_{d}\left(\lambda, \theta_{0}, N\right) \geq \bar{Q}_{d}, \\
& 0 \leq N \theta_{0} \leq B \quad N=1,2,3 \ldots
\end{array}
$$

Obviously, $\mathbb{R}_{0}\left(\lambda, \theta_{0}, N\right)>\mathbb{R}_{1}\left(\lambda, \theta_{0}, N\right)$. Therefore, the optimization problem of the average throughput of the UAV can be approximated as follows:

$$
\begin{array}{ll}
\max _{\theta_{0}, N} & \mathbb{R}_{A}\left(\theta_{0}, N\right)=\mathbb{R}_{0}\left(\lambda, \theta_{0}, N\right), \\
\text { s.t. } & Q_{d}\left(\lambda, \theta_{0}, N\right) \geq \bar{Q}_{d}, \\
& 0 \leq N \theta_{0} \leq B \quad N=1,2,3 \ldots
\end{array}
$$

The time for UAV to transmit $M$ bits of data to BS can be given by $T_{a}=\left(M / \mathbb{B R}_{A}\left(\theta_{0}, N\right)\right)$.

Firstly, the optimal value of $\lambda$ in this multiparameter optimization problem is analysed. It is assumed that $\theta_{0}$ and $N$ are fixed, and $\lambda=\lambda_{0}$ is the detection threshold such that $Q_{d}\left(\lambda_{0}, \theta_{0}, N\right)=\bar{Q}_{d}$. Then we may choose a detection threshold $\lambda=\lambda_{1}$, which satisfies the constraint condition $Q_{d}\left(\lambda_{1}, \theta_{0}, N\right) \geq \bar{Q}_{d}$, and the average throughput $\mathbb{R}_{A}\left(\lambda_{1}, \theta_{0}, N\right)$ can be achieved from (30), (31), and (33):

$$
\begin{aligned}
\mathbb{R}_{A}\left(\lambda_{1}, \theta_{0}, N\right) & =\mathbb{R}_{0}\left(\lambda_{1}, \theta_{0}, N\right) \leq \mathbb{R}_{0}\left(\lambda_{0}, \theta_{0}, N\right)=\frac{B-N \theta_{0}}{B} P_{r}(\mu=0)\left(1-Q_{f}\left(\lambda_{0}, \theta_{0}\right)\right) \pi_{0} \\
& =\frac{B-N \theta_{0}}{B} P_{r}(\mu=0)\left(1-\mathbb{Q}\left(\sqrt{2 \gamma+1} \mathbb{Q}^{-1}\left(1-\left(1-\bar{Q}_{d}\right)^{\frac{1}{N}}\right)+\gamma \sqrt{\theta_{0} \frac{R_{s} f_{s}}{v}}\right)\right)^{N} \pi_{0}
\end{aligned}
$$

Therefore, $\lambda_{0}$ is the optimal detection threshold. Then, the optimizations of $\theta_{0}$ and $N$ are considered when $Q_{d}=\bar{Q}_{d}$. In one frame, the sensing SNR $\gamma$ of all mini sensing radians is approximately the same [23], and the optimization problem (33) can be simplified as follows:

$$
\begin{array}{ll}
\max _{\theta_{0}, N} & \mathbb{R}_{A}\left(\theta_{0}, N\right)=\frac{B-N \theta_{0}}{B} P_{r}(\mu=0)\left(1-\mathbb{Q}\left(\varepsilon_{0}+\gamma \sqrt{\theta_{0} \frac{R_{s} f_{s}}{v}}\right)\right)^{N} \pi_{0}, \\
\text { s.t. } \quad & 0 \leq N \theta_{0} \leq B \quad N=1,2,3 \ldots,
\end{array}
$$

where $\varepsilon_{0}=\sqrt{2 \gamma+1} \mathbb{Q}^{-1}\left(1-\left(1-\bar{Q}_{d}\right)^{1 / N}\right)$. When $N$ is assumed to be fixed, we set $U=(B / N)$ to analyse the optimization problem (35), and the problem can be rewritten as follows:

$$
\begin{array}{ll}
\max _{\theta_{0}, N} & \mathbb{R}_{A}\left(\theta_{0}\right)=\left(1-\frac{\theta_{0}}{U}\right) P_{r}(\mu=0)\left(1-\mathbb{Q}\left(\varepsilon_{0}+\gamma \sqrt{\theta_{0} \frac{R_{s} f_{s}}{v}}\right)\right)^{N} \pi_{0}, \\
\text { s.t. } \quad & 0 \leq \theta_{0} \leq U,
\end{array}
$$

Similar to (22), the optimization problem (36) can also be proved to be convex.

Theorem 2. There exists only one optimal $\theta_{0} \in[0, U]$ that maximizes $\mathbb{R}_{A}\left(\theta_{0}\right)$.

Proof. The first derivative and the second derivative of $\mathbb{R}_{A}\left(\theta_{0}\right)$ with respect to $\theta_{0}$ are, respectively, derived as follows:

$$
\begin{aligned}
\frac{d \mathbb{R}_{A}\left(\theta_{0}\right)}{d \theta_{0}}= & \pi_{0} P_{r}(\mu=0)\left[-\frac{\left(1-P_{f}\left(\theta_{0}\right)\right)^{N}}{U}+N\left(1-\frac{\theta_{0}}{U}\right)\left(1-P_{f}\left(\theta_{0}\right)\right)^{N-1} \frac{d P_{f}\left(\theta_{0}\right)}{d \theta_{0}}\right] . \\
\frac{d^{2} \mathbb{R}_{A}\left(\theta_{0}\right)}{d^{2} \theta_{0}}= & \pi_{0} P_{r}(\mu=0)\left[\frac{2 N\left(1-P_{f}\left(\theta_{0}\right)\right)^{N-1}}{U}\left(\frac{d^{2} P_{f}\left(\theta_{0}\right)}{d \theta_{0}}\right)+\left(N\left(1-\frac{\theta_{0}}{U}\right)\left(1-P_{f}\left(\theta_{0}\right)\right)^{N-2}\right)\right. \\
& \left.\times\left((N-1)\left(\frac{d P_{f}\left(\theta_{0}\right)}{d \theta_{0}}\right)^{2}-\left(1-P_{f}\left(\theta_{0}\right)\right) \frac{d^{2} P_{f}\left(\theta_{0}\right)}{d \theta_{0}}\right)\right] .
\end{aligned}
$$

From (37) and (38), it can be obtained that $\left(d^{2} \mathbb{R}_{A}\right.$ $\left.\left(\theta_{0}\right) / d^{2} \theta_{0}\right)<0$ when $p_{f}\left(\theta_{0}\right) \leq 0.5$, which implies that $\mathbb{R}_{A}\left(\theta_{0}\right)$ is a concave function of $\theta_{0}$. The bound of $\left(d \mathbb{R}_{A}\left(\theta_{0}\right) / d \theta_{0}\right)$ with $\theta_{0} \in[0, U]$ can be achieved as $\lim _{\theta_{0} \longrightarrow 0}\left(d \mathbb{R}_{A}\right.$ $\left.\left(\theta_{0}\right) / d \theta_{0}\right)=+\infty$ and $\lim _{\theta_{0} \rightarrow U}\left(d \mathbb{R}_{A}\left(\theta_{0}\right) / d \theta_{0}\right)<0$, which indicates that there exists an optimal $\theta_{0} \in[0, U]$ defined as 


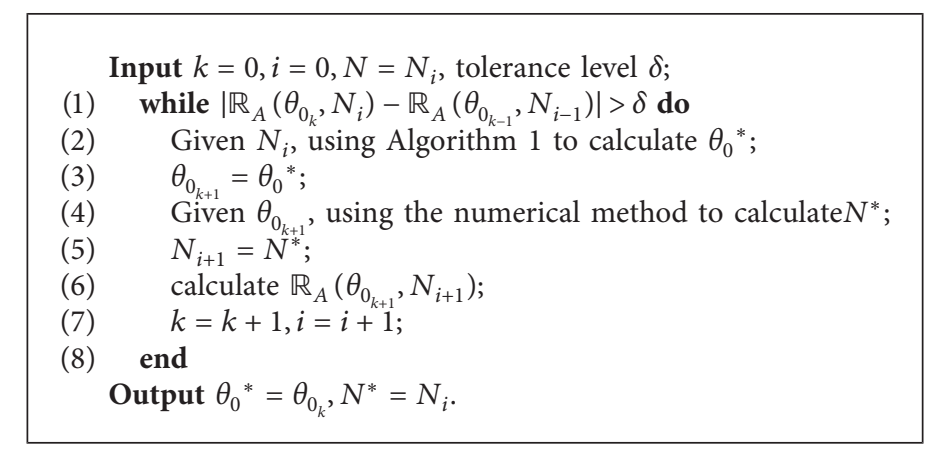

Algorithm 2: AIO (joint optimization of $\theta_{0}$ and $N$ ).

TABLE 1: Simulation parameters.

\begin{tabular}{|c|c|c|c|c|c|}
\hline Parameter & Value & Parameter & Value & Parameter & Value \\
\hline$R_{P}(\mathrm{~m})$ & 320 & B (rad) & $\pi / 3$ & $P_{r}(\mu=1)$ & 0.2 \\
\hline$R_{S}(\mathrm{~m})$ & 50 & $\omega_{1}$ & 9.6 & $\dot{\zeta}_{\mathrm{LoS}}$ & 3 \\
\hline$H(\mathrm{~m})$ & 60 & $\omega_{2}$ & 0.28 & $\zeta_{\underline{N L O S}}$ & 10 \\
\hline$f(\mathrm{kHz})$ & 500 & $f_{s}(\mathrm{kHz})$ & 60 & $\bar{P}_{d}$ & 0.9 \\
\hline$P_{S}(\mathrm{~W})$ & 10 & $P_{P}(\mathrm{~W})$ & 10 & $\bar{Q}_{d}$ & 0.9 \\
\hline$M$ (bit) & $10^{5}$ & $\mathbb{B}(\mathrm{kHz})$ & 20 & $l$ & 6 \\
\hline
\end{tabular}

$\theta_{0}^{*}$ that maximizes $\mathbb{R}_{A}$. Furthermore, Algorithm 1 can be used to obtain $\theta_{0}^{*}$.

When $\theta_{0}^{*}$ is achieved, the optimal number of sensing radians denoted as $N^{*}$ can be obtained by using the numerical method. Hence, the searching process of $N$ can be described as $N^{*}=\arg \max _{N=1,2, \ldots N_{\max }} \mathbb{R}_{A}\left(N, \theta_{0}\right)$, and it should be noted that $1 \leq N \leq\left\lceil B / \theta_{0}\right\rceil$, where $\lceil x\rceil$ denotes the smallest integer not less than $x$. In addition, the joint optimization of $\theta_{0}$ and $N$ can be realized by using the AIO algorithm, and the iteration terminates either when iteration times are reaching the maximum limit or when the accuracy of the throughput is less than the tolerance level. Details of the proposed AIO algorithm are given in Algorithm 2.

\section{Simulation Results}

The proposed optimization schemes of SE in both singleradian spectrum sensing and multiradian CSS are evaluated by numerical results in this section. The spectrum sensing performance of UAV is investigated in the UAV-based CRN. Other selected network parameters such as flight position and flight speed are considered in the following analysis. The simulation parameters are summarized in Table 1.

4.1. SE Optimization Scheme of Single Sensing Radian. In this section, we optimize the SE of UAV in single sensing radian and show that there exists an optimal sensing radian to maximize the average throughput $\mathbb{R}_{A}$ of UAV and minimize the transmission time $T_{a}$ of $M$ bits data. Firstly, the sensing scenario of UAV is static sensing in one frame, and the angle between UAV and $x$-axis is assumed to be $\alpha=0$ (rad). Figure 3 indicates $\mathbb{R}_{A}$ of UAV and $T_{a}$ changing with $\theta$ under different flight speeds.

As shown in Figure 3, with the increase of $\theta, \mathbb{R}_{A}$ increases rapidly at first and then decreases slowly after reaching the maximum denoted as $\mathbb{R}_{\mathrm{A}, \max }$. However, $T_{a}$ has opposite change with $\mathbb{R}_{A}$, and the minimum of data transmission time is denoted as $T_{a \text {,min }}$. The reason is that, with the increase of $\theta$, the sensing performance will be improved, but the transmission radian will be smaller if more sensing radians are consumed, and $\mathbb{R}_{A}$ will be consequently affected. Moreover, Figure 3 also demonstrates that $\theta^{*}$ and $T_{a \text {,min }}$ both increase with the flight speed, while $\mathbb{R}_{A, \max }$ decreases with the flight speed. As a result, a trade-off between $\mathbb{R}_{A}$ and $T_{a}$ should be achieved by optimizing the sensing radian.

When the sensing scenario of UAV is dynamic sensing, the angle $\alpha$ between UAV and $x$-axis changes periodically in the range of $[0,2 \pi]$ with the change of the UAV's position. In one flight cycle, the flight path and sensing performance of UAV are symmetric with respect to the $x$-axis. Figure 4 indicates $\mathbb{R}_{\mathrm{A} \text {,max }}$ and $T_{a \text {,min }}$ changing with the angle $\alpha$ under different flight speeds.

It can be seen that $T_{a \text {,min }}$ increases first and then decreases during the UAV's flight around the BS. When $\alpha=\pi$, that is, the distance between the UAV and the PU is the furthest, $T_{a \text {,min }}$ achieves the maximum value. The reason is that the optimal sensing radian increases first and then decreases with the change of $\alpha$ from 0 to $2 \pi$, and the corresponding transmission radian shows the opposite trend. Therefore, $\mathbb{R}_{\mathrm{A} \text {,max }}$ decreases first and then increases with the change of the UAV's position, and $T_{a \text {,min }}$ has opposite change with $\mathbb{R}_{A, \max }$. It can also be noticed that the increase of the flight speed will lead to the decrease of $\mathbb{R}_{\mathrm{A} \text {,max }}$, so that $T_{a \text {,min }}$ will be increased accordingly.

4.2. SE Optimization Scheme of Multiradian CSS. In this section, we optimize the SE of UAV under multiradian CSS and prove that there exist an optimal mini sensing radian denoted as $\theta_{0}^{*}$ and an optimal number of mini sensing radians 


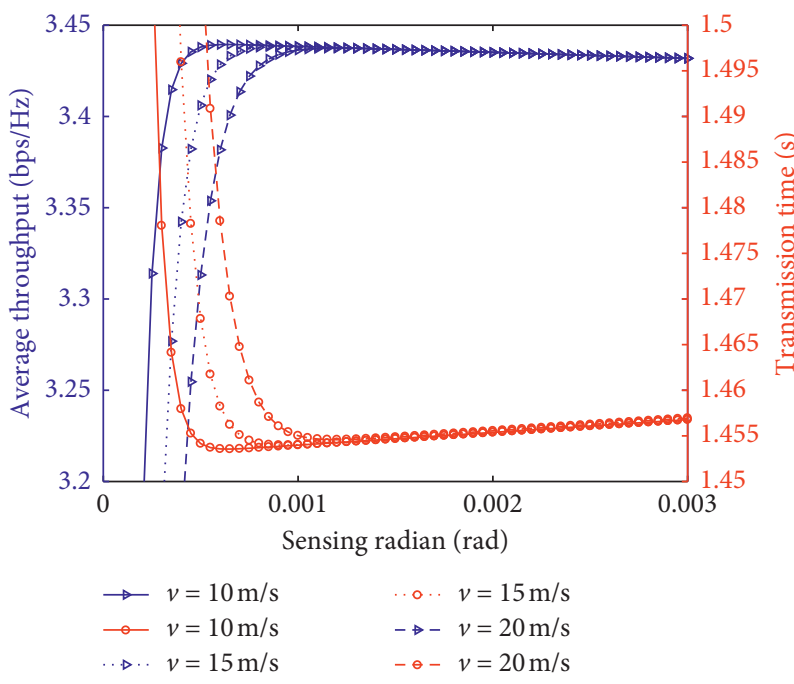

Figure 3: Average throughput and transmission time versus sensing radian under different flight speeds.

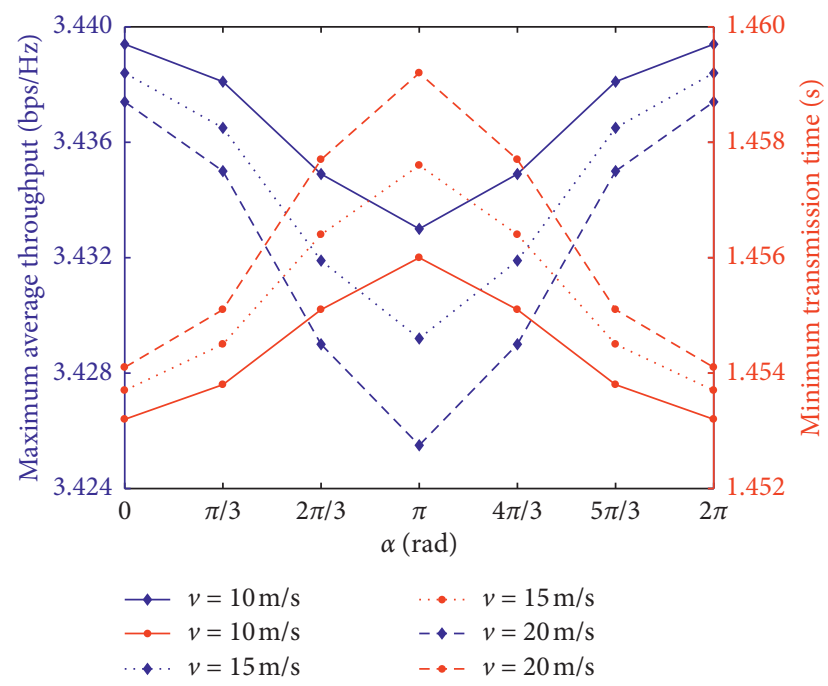

FIGURE 4: Maximal average throughput and minimal transmission time versus the angle $\alpha$ under different flight speeds.

denoted as $N^{*}$ to maximize the average throughput $\mathbb{R}_{A}$ of UAV and minimize the transmission time $T_{a}$ of $M$ bits data.

In one frame, the sensing scenario of UAV is static sensing, and it is assumed that the angle between UAV and $x$-axis is $\alpha=\pi(\mathrm{rad})$, and the UAV's flight speed is $v=20(\mathrm{~m} / \mathrm{s})$. Figure 5 depicts $\mathbb{R}_{A}$ and $T_{a}$ versus $N$ under different values of $\theta_{0}$. It can be seen from Figure 5 that there exists a unique $N^{*}$ to maximize $\mathbb{R}_{A}$ and minimize $T_{a}$, and it can also be noticed that $\mathbb{R}_{\mathrm{A}, \max }$ increases with $\theta_{0}$, while $T_{a \text {,min }}$ and $N^{*}$ decrease with the increase of $\theta_{0}$. Hence, the joint optimization algorithm proposed in Section 3 can be convergent after several iterations.

The performance differences between the proposed AIO algorithm and the SRO algorithm in [19] versus mini sensing radian in average spectrum efficiency are investigated in Figure 6. It can be seen that the proposed AIO algorithm has better performance than the SRO algorithm which only

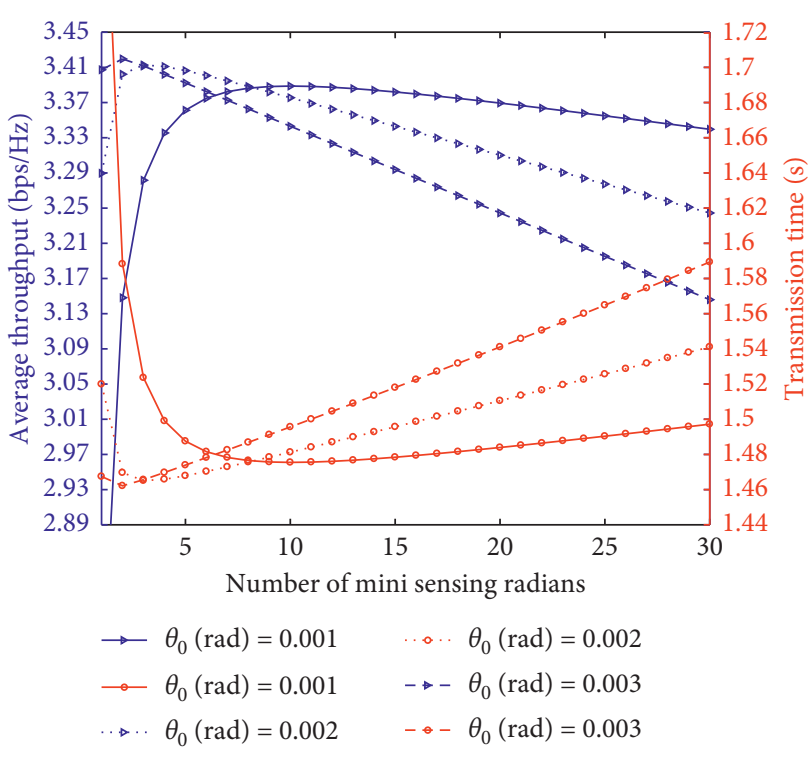

FIgURE 5: Average throughput and transmission time versus number of sensing radians under different mini sensing radian.

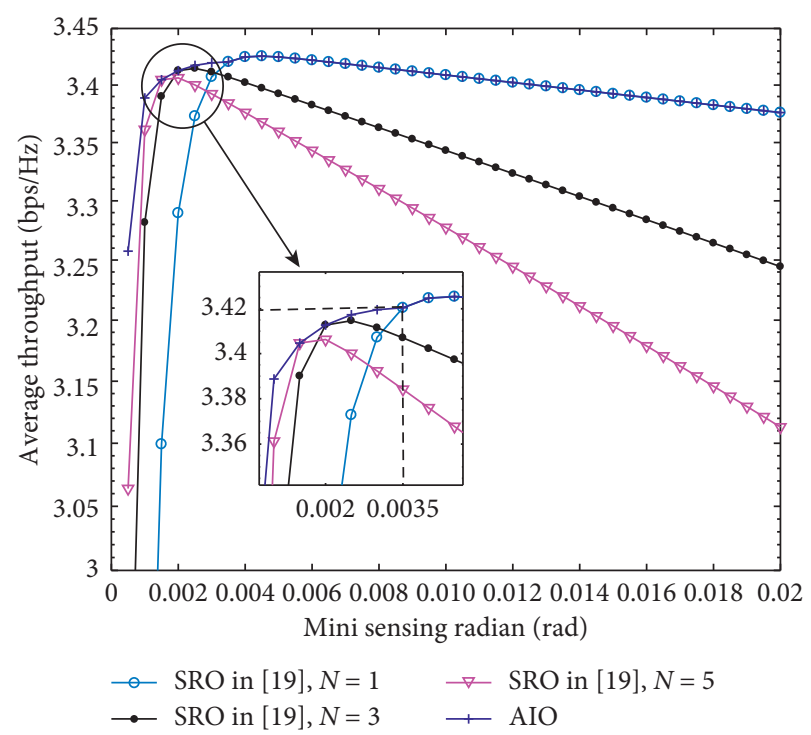

FIGURE 6: Average throughput versus mini sensing radian under different algorithm.

optimizes mini sensing radian with a fixed number of mini sensing radians, and it can also be noticed that $\mathbb{R}_{\mathrm{A}, \max }$ obtained by the SRO algorithm decreases with the increase of $N$. Moreover, the SRO algorithm with $N=1$ and the AIO algorithm achieve the same optimal SE when $\theta_{0}>0.0035 \mathrm{rad}$. Hence, it can be obtained from Figure 6 that the multiradian CSS method has application potential when $\theta_{0}<0.0035$, and the optimal number of mini sensing radians is $N^{*}=1$ when $\theta_{0}>0.0035$.

When the sensing scenario of UAV is dynamic sensing, the influence of UAV's flight path on the sensing SNR $\gamma$ is considered, and the flight path of the UAV is symmetrical with respect to the $x$-axis in one flight cycle. Figure 7 depicts $N^{*}$ versus $\theta_{0}$ when the UAV is in different frames in half a 


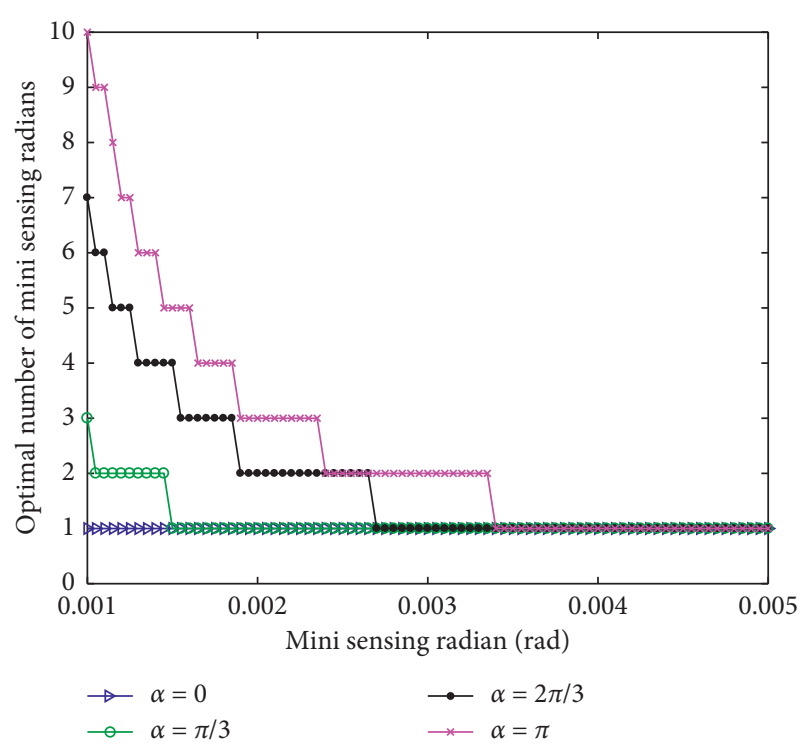

FIGURE 7: Optimal number of mini sensing radians versus mini sensing radian under different $\alpha$.

flight cycle. It can be seen from Figure 7 that, with the increase of $\theta_{0}, N^{*}$ that maximizes $\mathbb{R}_{A}$ shows a stepwise downward trend. It can also be noticed that when $\theta_{0}$ increases to a certain extent, the optimal number of mini sensing radians is $N^{*}=1$. The reason is that if the sensing radian is large enough, the multiradian CSS scheme is not needed to enhance the sensing performance.

The sensing SNR $\gamma$ in the UAV-based CRN decreases and $N^{*}$ increases with the change of $\alpha$ from 0 to $\pi$. Obviously, the distance between UAV and PU is the furthest when $\alpha=\pi$, and $\gamma$ is the smallest and $N^{*}$ is the largest at this time. Therefore, the multiradian CSS technology has more distinguished effects in improving the average throughput of the secondary link and ensuring the QoS of the PU when $\gamma$ is low.

\section{Conclusions}

In this paper, a UAV-based CRN is established to investigate the SE optimization of the UAV in A2G channel. The UAV flies circularly and performs spectrum sensing and data transmission in each frame. On the premise of ensuring the QoS of the PU, the sensing radian is optimized to maximize the throughput of the secondary UAV link, and the data transmission time of UAV to transmit $M$ bits of data to BS is optimized. In multiradian CSS, the AIO algorithm is proposed to jointly optimize the mini sensing radian and the number of mini sensing radians to maximize throughput and minimize the data transmission time. The simulation results show that, compared with the SRO algorithm, the proposed AIO algorithm can preferably solve the SE optimization problem of the secondary network, and the proposed multiradian CSS scheme has better performance to guarantee the QoS of the PU and improve the communication performance of the UAV compared with the singleradian spectrum sensing scheme, especially in the severe channel environments.

\section{Data Availability}

The data used to support the findings of this study are available from the corresponding author upon request.

\section{Conflicts of Interest}

The authors declare that there are no conflicts of interest regarding the publication of this paper.

\section{Acknowledgments}

The authors would like to thank Dr. Ruiyang Xu and Dr. Haobo Wang from the communication system laboratory of Air Force Engineering University for their support in the experiment and technology of this paper. This work was supported in part by the National Natural Science Foundation of China under Grant 61901509, Grant 61901490, and Grant 61571460, in part by the National Post-Doctoral Program for Innovative Talents under Grant BX201700108, and in part by the Research and Innovation Foundation of AFEU under Grant YNLX190025.

\section{References}

[1] H. Niu, P. N. Gonzalez, and R. W. Heath, "A UAV-based traffic monitoring system-invited paper," in Proceedings of the 2018 IEEE 87th Vehicular Technology Conference (VTC Spring), pp. 1-5, Porto, Portugal, June 2018.

[2] T. Samad, J. S. Bay, and D. Godbole, "Network-centric systems for military operations in urban terrain: the role of UAVs," Proceedings of the IEEE, vol. 95, no. 1, pp. 92-107, 2007.

[3] A. Jaimes, S. Kota, and J. Gomez, "An approach to surveillance an area using swarm of fixed wing and quad-rotor unmanned aerial vehicles UAV (s)," in Proceedings of the 2008 IEEE International Conference on System of Systems Engineering, pp. 1-6, Monterey, CA, USA, June 2008.

[4] M. Mozaffari, W. Saad, M. Bennis, and M. Debbah, "Drone small cells in the clouds: design, deployment and performance analysis," in Proceedings of the 2015 IEEE Global Communications Conference (GLOBECOM), pp. 1-6, San Diego, CA, USA, December 2015.

[5] K. Samdanis, X. Costa-Perez, and V. Sciancalepore, "From network sharing to multi-tenancy: the $5 G$ network slice broker," IEEE Communications Magazine, vol. 54, no. 7, pp. 32-39, 2016.

[6] A. Sultana, L. Zhao, and X. Fernando, "Energy-efficient power allocation in underlay and overlay cognitive device-to-device communications," IET Communications, vol. 13, no. 2, pp. 162-170, 2019.

[7] J. Gubbi, R. Buyya, S. Marusic, and M. Palaniswami, "Internet of things (IoT): a vision, architectural elements, and future directions," Future Generation Computer Systems, vol. 29, no. 7, pp. 1645-1660, 2013.

[8] Y. Saleem, M. H. Rehmani, and S. Zeadally, "Integration of cognitive radio technology with unmanned aerial vehicles: issues, opportunities, and future research challenges," Journal of Network and Computer Applications, vol. 50, pp. 15-31, 2015.

[9] H. Hu, X. Da, L. Ni, Y. Huang, and H. Zhang, "Green energy powered cognitive sensor network with cooperative sensing," IEEE Access, vol. 7, pp. 17354-17364, 2019. 
[10] W. Xu, S. Wang, S. Yan, and J. He, "An efficient wideband spectrum sensing algorithm for unmanned aerial vehicle communication networks," IEEE Internet of Things Journal, vol. 6, no. 2, pp. 1768-1780, 2019.

[11] Y. Pan, X. Da, H. Hu, Z. Zhu, R. Xu, and L. Ni, "Energyefficiency optimization of UAV-based cognitive radio system," IEEE Access, vol. 7, pp. 155381-155391, 2019.

[12] W. Na, J. Yoon, S. Cho, D. Griffith, and N. Golmie, "Centralized cooperative directional spectrum sensing for cognitive radio networks," IEEE Transactions on Mobile Computing, vol. 17, no. 6, pp. 1260-1274, 2018.

[13] C. Bhatnagar, A. Potnis, P. Dwivedy, and S. K. Meena, "Performance analysis and optimization schemes for cooperative spectrum sensing and information fusion for cognitive radio: a survey," in Proceedings of the 2017 1st International Conference on Electronics, Materials Engineering and Nano-Technology (IEMENTech), pp. 1-6, Kolkata, WB, India, April 2017.

[14] R. Kishore, S. Gurugopinath, S. Muhaidat, P. C. Sofotasios, O. A. Dobre, and N. Al-Dhahir, "Sensing-throughput tradeoff for superior selective reporting-based spectrum sensing in energy harvesting HCRNs," IEEE Transactions on Cognitive Communications and Networking, vol. 5, no. 2, pp. 330-341, 2019.

[15] G. M. D. Santana, R. S. Cristo, C. Dezan, J.-P. Diguet, D. P. M. Osorio, and K. R. L. J. C. Branco, "Cognitive radio for UAV communications: opportunities and future challenges," in Proceedings of the 2018 International Conference on Unmanned Aircraft Systems (ICUAS), pp. 760-768, Dallas, TX, USA, June 2018.

[16] L. Sboui, H. Ghazzai, Z. Rezki, and M.-S. Alouini, “Achievable rates of UAV-relayed cooperative cognitive radio MIMO systems," IEEE Access, vol. 5, pp. 5190-5204, 2017.

[17] Y. Zheng, Y. Wang, and F. Meng, "Modeling and simulation of pathloss and fading for air-ground link of HAPs within a network simulator," in Proceedings of the 2013 International Conference on Cyber-Enabled Distributed Computing and Knowledge Discovery, pp. 421-426, IEEE, Beijing, China, October 2013

[18] H. Hu, X. Da, Y. Huang, H. Zhang, L. Ni, and Y. Pan, "SE and EE optimization for cognitive UAV network based on location information," IEEE Access, vol. 7, pp. 162115-162126, 2019.

[19] X. Liu, M. Guan, X. Zhang, and H. Ding, "Spectrum sensing optimization in an UAV-based cognitive radio," IEEE Access, vol. 6, pp. 44002-44009, 2018.

[20] H. Ghazzai, M. Ben Ghorbel, A. Kadri, M. J. Hossain, and H. Menouar, "Energy-efficient management of unmanned aerial vehicles for underlay cognitive radio systems," IEEE Transactions on Green Communications and Networking, vol. 1, no. 4, pp. 434-443, 2017.

[21] A. Al-Hourani, S. Kandeepan, and S. Lardner, "Optimal lap altitude for maximum coverage," IEEE Wireless Communications Letters, vol. 3, no. 6, pp. 569-572, 2014.

[22] Y.-C. Liang, Y. Zeng, E. C. Y. Peh, and A. T. Hoang, "Sensingthroughput tradeoff for cognitive radio networks," IEEE Transactions on Wireless Communications, vol. 7, no. 4, pp. 1326-1337, 2008.

[23] L. Liu, S. Zhang, and R. Zhang, "CoMP in the sky: UAV placement and movement optimization for multi-user communications," IEEE Transactions on Communications, vol. 67, no. 8, pp. 5645-5658, 2019. 